Kragujevac Journal of Mathematics

Volume 44(4) (2020), Pages 509-522.

\title{
SHARP BOUNDS ON THE AUGMENTED ZAGREB INDEX OF GRAPH OPERATIONS
}

\author{
N. DEHGARDI ${ }^{1}$ AND H. ARAM AR $^{2 *}$
}

Abstract. Let $G$ be a finite and simple graph with edge set $E(G)$. The augmented Zagreb index of $G$ is

$$
A Z I(G)=\sum_{u v \in E(G)}\left(\frac{d_{G}(u) d_{G}(v)}{d_{G}(u)+d_{G}(v)-2}\right)^{3},
$$

where $d_{G}(u)$ denotes the degree of a vertex $u$ in $G$. In this paper, we give some bounds of this index for join, corona, cartesian and composition product of graphs by general sum-connectivity index and general Randić index and compute the sharp amount of that for the regular graphs.

\section{INTRODUCTION}

Let $G$ be a finite and simple graph with vertex set $V=V(G)$ and edge set $E=E(G)$. The integers $n=n(G)=|V(G)|$ and $m=m(G)=|E(G)|$ are the order and the size of the graph $G$, respectively. For a vertex $v \in V(G)$, the open neighborhood of $v$, denoted by $N_{G}(v)=N(v)$, is the set $\{u \in V(G) \mid u v \in E(G)\}$. The degree of $v \in V(G)$, denoted by $d_{G}(v)$, is defined by $d_{G}(v)=\left|N_{G}(v)\right|$. The maximum (resp. minimum) degree of vertices of $G$ is denoted by $\Delta_{G}$ (resp. $\delta_{G}$ ). We use Bondy and Murty [10] for terminology and notation not defined here.

Several authors defined and studied more vertex degree-based graph invariants such as [16]. One of them is augmented Zagreb index of $G$ that is proposed in 2010 by Furtula et al. [15] as

Key words and phrases. Augmented Zagreb index, general sum-connectivity index, general Randić index, graph operations.

2010 Mathematics Subject Classification. Primary: 05C12. Secondary: 05C07.

DOI 10.46793/KgJMat2004.509D

Received: December 02, 2017.

Accepted: June 13, 2018. 


$$
A Z I(G)=\sum_{u v \in E(G)}\left(\frac{d_{G}(u) d_{G}(v)}{d_{G}(u)+d_{G}(v)-2}\right)^{3},
$$

where $d_{G}(u)$ denotes the degree of a vertex $u$ in $G$. The researchers give a good bounds for it by using different graph parameters, investigate the impact of removing and adding the edge for graph on the augmented Zagreb index. For details see [1,18,24,27].

In 2009, $\mathrm{Zu}$ and Trijnastić [28] defined the sum-connectivity index as

$$
\chi(G)=\sum_{u v \in E(G)} d_{G}(u)+d_{G}(v)
$$

and one year later, they in [29] introduced the general sum-connectivity index as

$$
\chi_{\lambda}(G)=\sum_{u v \in E(G)}\left(d_{G}(u)+d_{G}(v)\right)^{\lambda}, \quad \text { for } \lambda \in \mathbb{R} .
$$

There are good results on general sum-connectivity index such as [22,23]. In 1975, the chemist Milan Randić [21] introduced a topological index $R(G)$ under the name branching index. The branching index was renamed the molecular connectivity index and is often referred to as the Randic index and later named second Zagreb index. In 1998, Bollobas and Erdos [9] proposed the generealization state of it named general Randić index, $R_{\lambda}(G)$, as

$$
R_{\lambda}(G)=\sum_{u v \in E(G)}\left(d_{G}(u) d_{G}(v)\right)^{\lambda}, \quad \text { for } \lambda \in \mathbb{R}
$$

later that is named second general Zagreb index.

The relation between several indices and operations of graphs were very studied. (see $[2-8,11-14,17,19,20,25,26])$. In this paper, we calculate bounds of the augmented Zagreb index by two other indices, the general sum-connectivity index and the Randic index for join, corona, cartesian and composition product of graphs and compute the sharp amount of that for the regular graphs.

\section{The Join OF Graphs}

The join $G+H$ of graphs $G$ and $H$ with disjoint vertex sets $V(G)$ and $V(H)$ and edge sets $E(G)$ and $E(H)$ is the graph union $G \cup H$ together with all the edges joining $V(G)$ and $V(H)$. Obviously, $|V(G+H)|=|V(G)|+|V(H)|$ and $|E(G+H)|=|E(G)|+|E(H)|+|V(G)||V(H)|$.

Theorem 2.1. Let $G$ be a graph of order $n_{1}$ and of size $m_{1}$ and let $H$ be a graph of order $n_{2}$ and of size $m_{2}$. Then

$$
\begin{aligned}
A Z I(G+H) \leq & \frac{\left(\Delta_{G}-1\right)^{3} A Z I(G)}{\left(\Delta_{G}+n_{2}-1\right)^{3}}+\frac{n_{2}^{3} \chi_{3}(G)+\left(3 n_{2}^{2} \Delta_{G}^{2}+3 n_{2}^{4}\right) \chi_{2}(G)+3 n_{2}^{5} \chi_{1}(G)}{8\left(\delta_{G}+n_{2}-1\right)^{3}} \\
& +\frac{\left(6 n_{2} \Delta_{G}+3 n_{2}^{2}\right) R_{2}(G)+\left(12 n_{2}^{3} \Delta_{G}+3 n_{2}^{4}\right) R_{1}(G)+m_{1} n_{2}^{6}}{8\left(\delta_{G}+n_{2}-1\right)^{3}}
\end{aligned}
$$




$$
\begin{aligned}
& +\frac{\left(\Delta_{H}-1\right)^{3} A Z I(H)}{\left(\Delta_{H}+n_{1}-1\right)^{3}} \\
& +\frac{n_{1}^{3} \chi_{3}(H)+\left(3 n_{1}^{2} \Delta_{H}^{2}+3 n_{1}^{4}\right) \chi_{2}(H)+3 n_{1}^{5} \chi_{1}(H)}{8\left(\delta_{H}+n_{1}-1\right)^{3}} \\
& +\frac{\left(6 n_{1} \Delta_{H}+3 n_{1}^{2}\right) R_{2}(H)+\left(12 n_{1}^{3} \Delta_{H}+3 n_{1}^{4}\right) R_{1}(H)+m_{2} n_{1}^{6}}{8\left(\delta_{H}+n_{1}-1\right)^{3}} \\
& +n_{1} n_{2}\left(\frac{\left(\Delta_{G}+n_{2}\right)\left(\Delta_{H}+n_{1}\right)}{\delta_{G}+\delta_{H}+n_{1}+n_{2}-2}\right)^{3},
\end{aligned}
$$

with equality if and only if $G$ and $H$ are regular graphs.

Proof. By definition,

$$
A Z I(G+H)=\sum_{u v \in E(G+H)}\left(\frac{d_{G+H}(u) d_{G+H}(v)}{d_{G+H}(u)+d_{G+H}(v)-2}\right)^{3} .
$$

We partition the edges of $G+H$ in to three subset $E_{1}, E_{2}$ and $E_{3}$, as follows:

$$
\begin{aligned}
& E_{1}=\{e=u v \mid u, v \in V(G)\}, \\
& E_{2}=\{e=u v \mid u, v \in V(H)\}, \\
& E_{3}=\{e=u v \mid u \in V(G), v \in V(H)\} .
\end{aligned}
$$

Let $e=u v \in E_{1}$. Then $d_{G+H}(u)=d_{G}(u)+n_{2}$ and $d_{G+H}(v)=d_{G}(v)+n_{2}$. Hence

$$
\begin{aligned}
\left(\left(d_{G}(u)+n_{2}\right)\left(d_{G}(v)+n_{2}\right)\right)^{3}= & \left(d_{G}(u) d_{G}(v)\right)^{2}\left[3 n_{2}\left(d_{G}(u)+d_{G}(v)\right)+3 n_{2}^{2}\right] \\
& +\left(d_{G}(u) d_{G}(v)\right)^{3}+d_{G}(u) d_{G}(v) \\
& \times\left[6 n_{2}^{3}\left(d_{G}(u)+d_{G}(v)\right)+3 n_{2}^{4}\right] \\
& +n_{2}^{3}\left(d_{G}(u)+d_{G}(v)\right)^{3}+3 n_{2}^{5}\left(d_{G}(u)+d_{G}(v)\right) \\
& +\left(d_{G}(u)+d_{G}(v)\right)^{2}\left[3 n_{2}^{2} d_{G}(u) d_{G}(v)+3 n_{2}^{4}\right]+n_{2}^{6}
\end{aligned}
$$

and

$$
\begin{aligned}
& \left(\frac{d_{G+H}(u) d_{G+H}(v)}{d_{G+H}(u)+d_{G+H}(v)-2}\right)^{3} \\
= & \left(1-\frac{2 n_{2}}{d_{G}(u)+d_{G}(v)+2 n_{2}-2}\right)^{3}\left(\frac{d_{G}(u) d_{G}(v)}{d_{G}(u)+d_{G}(v)-2}\right)^{3} \\
& +\frac{n_{2}^{3}\left(d_{G}(u)+d_{G}(v)\right)^{3}+\left[3 n_{2}^{2}\left(d_{G}(u) d_{G}(v)\right)^{2}+3 n_{2}^{4}\right]\left(d_{G}(u)+d_{G}(v)\right)^{2}}{\left(d_{G}(u)+d_{G}(v)+2 n_{2}-2\right)^{3}} \\
& +\frac{3 n_{2}^{5}\left(d_{G}(u)+d_{G}(v)\right)+\left[3 n_{2}\left(d_{G}(u)+d_{G}(v)\right)+3 n_{2}^{2}\right]\left(d_{G}(u) d_{G}(v)\right)^{2}}{\left(d_{G}(u)+d_{G}(v)+2 n_{2}-2\right)^{3}} \\
& +\frac{\left[6 n_{2}^{3}\left(d_{G}(u)+d_{G}(v)\right)+3 n_{2}^{4}\right] d_{G}(u) d_{G}(v)+n_{2}^{6}}{\left(d_{G}(u)+d_{G}(v)+2 n_{2}-2\right)^{3}}
\end{aligned}
$$




$$
\begin{aligned}
\leq & \left(\frac{\Delta_{G}-1}{\Delta_{G}+n_{2}-1}\right)^{3}\left(\frac{d_{G}(u) d_{G}(v)}{d_{G}(u)+d_{G}(v)-2}\right)^{3} \\
& +\frac{n_{2}^{3}\left(d_{G}(u)+d_{G}(v)\right)^{3}+\left(3 n_{2}^{2} \Delta_{G}^{2}+3 n_{2}^{4}\right)\left(d_{G}(u)+d_{G}(v)\right)^{2}}{8\left(\delta_{G}+n_{2}-1\right)^{3}} \\
& +\frac{3 n_{2}^{5}\left(d_{G}(u)+d_{G}(v)\right)+\left(6 n_{2} \Delta_{G}+3 n_{2}^{2}\right)\left(d_{G}(u) d_{G}(v)\right)^{2}}{8\left(\delta_{G}+n_{2}-1\right)^{3}} \\
& +\frac{\left(12 n_{2}^{3} \Delta_{G}+3 n_{2}^{4}\right) d_{G}(u) d_{G}(v)+n_{2}^{6}}{8\left(\delta_{G}+n_{2}-1\right)^{3}} .
\end{aligned}
$$

Therefore,

$$
\begin{aligned}
\sum_{u v \in E_{1}}\left(\frac{d_{G+H}(u) d_{G+H}(v)}{d_{G+H}(u)+d_{G+H}(v)-2}\right)^{3} \leq & \left(\frac{\Delta_{G}-1}{\Delta_{G}+n_{2}-1}\right)^{3} A Z I(G)+\frac{m_{1} n_{2}^{6}}{8\left(\delta_{G}+n_{2}-1\right)^{3}} \\
& +\frac{n_{2}^{3} \chi_{3}(G)+\left(3 n_{2}^{2} \Delta_{G}^{2}+3 n_{2}^{4}\right) \chi_{2}(G)+n_{2}^{5} \chi_{1}(G)}{8\left(\delta_{G}+n_{2}-1\right)^{3}} \\
& +\frac{\left(6 n_{2} \Delta_{G}+3 n_{2}^{2}\right) R_{2}(G)+\left(12 n_{2}^{3} \Delta_{G}+3 n_{2}^{4}\right) R_{1}(G)}{8\left(\delta_{G}+n_{2}-1\right)^{3}} .
\end{aligned}
$$

Obviously, equality holds if and only if $\Delta_{G}=\delta_{G}$. Similarly

$$
\begin{aligned}
\sum_{u v \in E_{2}}\left(\frac{d_{G+H}(u) d_{G+H}(v)}{d_{G+H}(u)+d_{G+H}(v)-2}\right)^{3} \leq & \left(\frac{\Delta_{H}-1}{\Delta_{H}+n_{1}-1}\right)^{3} A Z I(H)+\frac{m_{2} n_{1}^{6}}{8\left(\delta_{H}+n_{1}-1\right)^{3}} \\
& +\frac{n_{1}^{3} \chi_{3}(H)+\left(3 n_{1}^{2} \Delta_{H}^{2}+3 n_{1}^{4}\right) \chi_{2}(H)+3 n_{1}^{5} \chi_{1}(H)}{8\left(\delta_{H}+n_{1}-1\right)^{3}} \\
& +\frac{\left(6 n_{1} \Delta_{H}+3 n_{1}^{2}\right) R_{2}(H)+\left(12 n_{1}^{3} \Delta_{H}+3 n_{1}^{4}\right) R_{1}(H)}{8\left(\delta_{H}+n_{1}-1\right)^{3}} .
\end{aligned}
$$

Equality holds if and only if $\Delta_{H}=\delta_{H}$. Let $e=u v \in E_{3}$ such that $u \in V(G)$ and $v \in V(H)$. Then $d_{G+H}(u)=d_{G}(u)+n_{2}$ and $d_{G+H}(v)=d_{H}(v)+n_{1}$. Hence for every edge $e=u v \in E_{3}$,

$$
\begin{aligned}
\left(\frac{d_{G+H}(u) d_{G+H}(v)}{d_{G+H}(u)+d_{G+H}(v)}\right)^{3} & =\left(\frac{\left(d_{G}(u)+n_{2}\right)\left(d_{H}(v)+n_{1}\right)}{d_{G}(u)+d_{H}(v)+n_{1}+n_{2}-2}\right)^{3} \\
& \leq\left(\frac{\left(\Delta_{G}+n_{2}\right)\left(\Delta_{H}+n_{1}\right)}{\delta_{G}+\delta_{H}+n_{1}+n_{2}-2}\right)^{3}
\end{aligned}
$$

Therefore,

$$
\sum_{u v \in E_{3}}\left(\frac{d_{G+H}(u) d_{G+H}(v)}{d_{G+H}(u)+d_{G+H}(v)}\right)^{3} \leq n_{1} n_{2}\left(\frac{\left(\Delta_{G}+n_{2}\right)\left(\Delta_{H}+n_{1}\right)}{\delta_{G}+\delta_{H}+n_{1}+n_{2}-2}\right)^{3},
$$


with equality if and only if $\Delta_{G}=\delta_{G}$ and $\Delta_{H}=\delta_{H}$. By Equations (2.1), (2.2) and (2.3), we have:

$$
\begin{aligned}
A Z I(G+H) \leq & \frac{\left(\Delta_{G}-1\right)^{3} A Z I(G)}{\left(\Delta_{G}+n_{2}-1\right)^{3}}+\frac{n_{2}^{3} \chi_{3}(G)+\left(3 n_{2}^{2} \Delta_{G}^{2}+3 n_{2}^{4}\right) \chi_{2}(G)+3 n_{2}^{5} \chi_{1}(G)}{8\left(\delta_{G}+n_{2}-1\right)^{3}} \\
& +\frac{\left(6 n_{2} \Delta_{G}+3 n_{2}^{2}\right) R_{2}(G)+\left(12 n_{2}^{3} \Delta_{G}+3 n_{2}^{4}\right) R_{1}(G)+m_{1} n_{2}^{6}}{8\left(\delta_{G}+n_{2}-1\right)^{3}} \\
& +\frac{\left(\Delta_{H}-1\right)^{3} A Z I(H)}{\left(\Delta_{H}+n_{1}-1\right)^{3}}+\frac{n_{1}^{3} \chi_{3}(H)+\left(3 n_{1}^{2} \Delta_{H}^{2}+3 n_{1}^{4}\right) \chi_{2}(H)+3 n_{1}^{5} \chi_{1}(H)}{8\left(\delta_{H}+n_{1}-1\right)^{3}} \\
& +\frac{\left(6 n_{1} \Delta_{H}+3 n_{1}^{2}\right) R_{2}(H)+\left(12 n_{1}^{3} \Delta_{H}+3 n_{1}^{4}\right) R_{1}(H)+m_{2} n_{1}^{6}}{8\left(\delta_{H}+n_{1}-1\right)^{3}} \\
& +n_{1} n_{2}\left(\frac{\left(\Delta_{G}+n_{2}\right)\left(\Delta_{H}+n_{1}\right)}{\delta_{G}+\delta_{H}+n_{1}+n_{2}-2}\right)^{3} .
\end{aligned}
$$

Equality holds if and only if $G$ and $H$ are regular graphs.

Theorem 2.2. Let $G$ be a graph of order $n_{1}$ and of size $m_{1}$ and let $H$ be a graph of order $n_{2}$ and of size $m_{2}$. Then

$$
\begin{aligned}
A Z I(G+H) \geq & \frac{\left(\delta_{G}-1\right)^{3} A Z I(G)}{\left(\delta_{G}+n_{2}-1\right)^{3}}+\frac{n_{2}^{3} \chi_{3}(G)+\left(3 n_{2}^{2} \delta_{G}^{2}+3 n_{2}^{4}\right) \chi_{2}(G)+3 n_{2}^{5} \chi_{1}(G)}{8\left(\Delta_{G}+n_{2}-1\right)^{3}} \\
& +\frac{\left(6 n_{2} \delta_{G}+3 n_{2}^{2}\right) R_{2}(G)+\left(12 n_{2}^{3} \delta_{G}+3 n_{2}^{4}\right) R_{1}(G)+m_{1} n_{2}^{6}}{8\left(\Delta_{G}+n_{2}-1\right)^{3}} \\
& +\frac{\left(\delta_{H}-1\right)^{3} A Z I(H)}{\left(\delta_{H}+n_{1}-1\right)^{3}}+\frac{n_{1}^{3} \chi_{3}(H)+\left(3 n_{1}^{2} \delta_{H}^{2}+3 n_{1}^{4}\right) \chi_{2}(H)+3 n_{1}^{5} \chi_{1}(H)}{8\left(\Delta_{H}+n_{1}-1\right)^{3}} \\
& +\frac{\left(6 n_{1} \delta_{H}+3 n_{1}^{2}\right) R_{2}(H)+\left(12 n_{1}^{3} \delta_{H}+3 n_{1}^{4}\right) R_{1}(H)+m_{2} n_{1}^{6}}{8\left(\Delta_{H}+n_{1}-1\right)^{3}} \\
& +n_{1} n_{2}\left(\frac{\left(\delta_{G}+n_{2}\right)\left(\delta_{H}+n_{1}\right)}{\Delta_{G}+\Delta_{H}+n_{1}+n_{2}-2}\right)^{3},
\end{aligned}
$$

with equality if and only if $G$ and $H$ are regular graphs.

Proof. Using an argument similar to that described in proof of Theorem 2.1, we obtained the result.

Corollary 2.1. Let $G$ be a k-regular graph of order $n_{1}$ and let $H$ be a r-regular graph of order $n_{2}$. Then

$$
A Z I(G+H)=\frac{k\left(k+n_{2}\right)^{6}}{16\left(k+n_{2}-1\right)^{3}}+\frac{r\left(r+n_{1}\right)^{6}}{16\left(r+n_{1}-1\right)^{3}}+\frac{n_{1} n_{2}\left(k+n_{2}\right)^{3}\left(r+n_{1}\right)^{3}}{\left(k+r+n_{1}+n_{2}-2\right)^{3}} .
$$

\section{The Corona Product of Graphs}

The corona product $G \circ H$ of graphs $G$ and $H$ with disjoint vertex sets $V(G)$ and $V(H)$ and edge sets $E(G)$ and $E(H)$ is as the graph obtained by taking one copy 
of $G$ and $|V(G)|$ copies of $H$ and joining the $i$-th vertex of $G$ to every vertex in $i$-th copy of $H$. Obviously, $|V(G \circ H)|=|V(G)|+|V(G)||V(H)|$ and $|E(G \circ H)|=$ $|E(G)|+|V(G)||E(H)|+|V(G)||V(H)|$.

Theorem 3.1. Let $G$ be a graph of order $n_{1}$ and of size $m_{1}$ and let $H$ be a graph of order $n_{2}$ and of size $m_{2}$. Then

$$
\begin{aligned}
A Z I(G \circ H) \leq & \frac{\left(\Delta_{G}-1\right)^{3} A Z I(G)}{\left(\Delta_{G}+n_{2}-1\right)^{3}}+\frac{n_{2}^{3} \chi_{3}(G)+\left(3 n_{2}^{2} \Delta_{G}^{2}+3 n_{2}^{4}\right) \chi_{2}(G)+3 n_{2}^{5} \chi_{1}(G)}{8\left(\delta_{G}+n_{2}-1\right)^{3}} \\
& +\frac{\left(6 n_{2} \Delta_{G}+3 n_{2}^{2}\right) R_{2}(G)+\left(12 n_{2}^{3} \Delta_{G}+3 n_{2}^{4}\right) R_{1}(G)+m_{1} n_{2}^{6}}{8\left(\delta_{G}+n_{2}-1\right)^{3}} \\
& +\frac{\left(\Delta_{H}-1\right)^{3} A Z I(H)}{\Delta_{H}^{3}}+\frac{\chi_{3}(H)+\left(3 \Delta_{H}^{2}+3\right) \chi_{2}(H)+3 \chi_{1}(H)}{8 \delta_{H}^{3}} \\
& +\frac{\left(6 \Delta_{H}+3\right) R_{2}(H)+\left(12 \Delta_{H}+3\right) R_{1}(H)+m_{2}}{8 \delta_{H}^{3}} \\
& +n_{1} n_{2}\left(\frac{\left(\Delta_{G}+n_{2}\right)\left(\Delta_{H}+1\right)}{\delta_{G}+\delta_{H}+n_{2}-1}\right)^{3}
\end{aligned}
$$

with equality if and only if $G$ and $H$ are regular graphs.

Proof. We partition the edges of $G$ in to three subset $E_{1}, E_{2}$ and $E_{3}$ such that $E_{1}=\{e=u v \mid u, v \in V(G)\}, E_{2}=\{e=u v \mid u, v \in V(H)\}$ and $E_{3}=\{e=u v \mid u \in$ $V(G), v \in V(H)\}$.

If $e=u v \in E_{1}$, then $d_{G \circ H}(u)=d_{G}(u)+n_{2}$ and $d_{G \circ H}(v)=d_{G}(v)+n_{2}$ and if $e=u v \in E_{2}$, then $d_{G \circ H}(u)=d_{H}(u)+1$ and $d_{G \circ H}(v)=d_{H}(v)+1$. By used of proof of Theorem 2.1, we have,

$$
\begin{aligned}
\sum_{u v \in E_{1}}\left(\frac{d_{G \circ H}(u) d_{G \circ H}(v)}{d_{G \circ H}(u)+d_{G \circ H}(v)-2}\right)^{3} \leq & \frac{\left(\Delta_{G}-1\right)^{3} A Z I(G)}{\left(\Delta_{G}+n_{2}-1\right)^{3}} \\
& +\frac{n_{2}^{3} \chi_{3}(G)+\left(3 n_{2}^{2} \Delta_{G}^{2}+3 n_{2}^{4}\right) \chi_{2}(G)+n_{2}^{5} \chi_{1}(G)}{8\left(\delta_{G}+n_{2}-1\right)^{3}} \\
& +\frac{\left(6 n_{2} \Delta_{G}+3 n_{2}^{2}\right) R_{2}(G)+\left(12 n_{2}^{3} \Delta_{G}+3 n_{2}^{4}\right) R_{1}(G)}{8\left(\delta_{G}+n_{2}-1\right)^{3}} \\
& +\frac{m_{1} n_{2}^{6}}{8\left(\delta_{G}+n_{2}-1\right)^{3}}, \\
(3.1) \quad & +\frac{\left(\Delta_{H}-1\right)^{3} A Z I(H)}{\Delta_{H}^{3}} \\
\sum_{u v \in E_{2}}\left(\frac{d_{G \circ H}(u) d_{G \circ H}(v)}{d_{G \circ H}(u)+d_{G \circ H}(v)-2}\right)^{3} \leq & +\frac{\left(6 \Delta_{H h}+3\right) R_{2}(H)+\left(12 \Delta_{H}+3\right) R_{1}(H)+m_{2}}{8 \delta_{H}^{3}} .
\end{aligned}
$$


Obviously, equalities hold if and only if $\Delta_{G}=\delta_{G}$ and $\Delta_{H}=\delta_{H}$.

Let $e=u v \in E_{3}$ such that $u \in V(G)$ and $v \in V(H)$. Then $d_{G \circ H}(u)=d_{G}(u)+n_{2}$ and $d_{G \circ H}(v)=d_{H}(v)+1$. Hence for every edge $e=u v \in E_{3}$,

$$
\begin{aligned}
\left(\frac{d_{G \circ H}(u) d_{G \circ H}(v)}{d_{G \circ H}(u)+d_{G \circ H}(v)-2}\right)^{3} & =\left(\frac{\left(d_{G}(u)+n_{2}\right)\left(d_{H}(v)+1\right)}{d_{G}(u)+d_{H}(v)+n_{2}+1-2}\right)^{3} \\
& \leq\left(\frac{\left(\Delta_{G}+n_{2}\right)\left(\Delta_{H}+1\right)}{\delta_{G}+\delta_{H}+n_{2}-1}\right)^{3} .
\end{aligned}
$$

Therefore,

$$
\sum_{u v \in E_{3}}\left(\frac{d_{G \circ H}(u) d_{G \circ H}(v)}{d_{G \circ H}(u)+d_{G \circ H}(v)-2}\right)^{3} \leq \frac{n_{1} n_{2}\left(\Delta_{G}+n_{2}\right)^{3}\left(\Delta_{H}+1\right)^{3}}{\left(\delta_{G}+\delta_{H}+n_{2}-1\right)^{3}},
$$

with equality if and only if $\Delta_{G}=\delta_{G}$ and $\Delta_{H}=\delta_{H}$. By Equations (3.1), (3.2) and (3.3), we have:

$$
\begin{aligned}
A Z I(G \circ H) \leq & \frac{\left(\Delta_{G}-1\right)^{3} A Z I(G)}{\left(\Delta_{G}+n_{2}-1\right)^{3}}+\frac{n_{2}^{3} \chi_{3}(G)+\left(3 n_{2}^{2} \Delta_{G}^{2}+3 n_{2}^{4}\right) \chi_{2}(G)+3 n_{2}^{5} \chi_{1}(G)}{8\left(\delta_{G}+n_{2}-1\right)^{3}} \\
& +\frac{\left(6 n_{2} \Delta_{G}+3 n_{2}^{2}\right) R_{2}(G)+\left(12 n_{2}^{3} \Delta_{G}+3 n_{2}^{4}\right) R_{1}(G)+m_{1} n_{2}^{6}}{8\left(\delta_{G}+n_{2}-1\right)^{3}} \\
& +\frac{\left(\Delta_{H}-1\right)^{3} A Z I(H)}{\Delta_{H}^{3}}+\frac{\chi_{3}(H)+\left(3 \Delta_{H}^{2}+3\right) \chi_{2}(H)+3 \chi_{1}(H)}{8 \delta_{H}^{3}} \\
& +\frac{\left(6 \Delta_{H}+3\right) R_{2}(H)+\left(12 \Delta_{H}+3\right) R_{1}(H)+m_{2}}{8 \delta_{H}^{3}} \\
& +n_{1} n_{2}\left(\frac{\left(\Delta_{G}+n_{2}\right)\left(\Delta_{H}+1\right)}{\delta_{G}+\delta_{H}+n_{2}-1}\right)^{3} .
\end{aligned}
$$

Equality holds if and only if $G$ and $H$ are regular graphs.

Theorem 3.2. Let $G$ be a graph of order $n_{1}$ and of size $m_{1}$ and let $H$ be a graph of order $n_{2}$ and of size $m_{2}$. Then

$$
\begin{aligned}
A Z I(G \circ H) \geq & \frac{\left(\delta_{G}-1\right)^{3} A Z I(G)}{\left(\delta_{G}+n_{2}-1\right)^{3}}+\frac{n_{2}^{3} \chi_{3}(G)+\left(3 n_{2}^{2} \delta_{G}^{2}+3 n_{2}^{4}\right) \chi_{2}(G)+3 n_{2}^{5} \chi_{1}(G)}{8\left(\Delta_{G}+n_{2}-1\right)^{3}} \\
& +\frac{\left(6 n_{2} \delta_{G}+3 n_{2}^{2}\right) R_{2}(G)+\left(12 n_{2}^{3} \delta_{G}+3 n_{2}^{4}\right) R_{1}(G)+m_{1} n_{2}^{6}}{8\left(\Delta_{G}+n_{2}-1\right)^{3}} \\
& +\frac{\left(\delta_{H}-1\right)^{3} A Z I(H)}{\delta_{H}^{3}}+\frac{\chi_{3}(H)+\left(3 \delta_{H}^{2}+3\right) \chi_{2}(H)+3 \chi_{1}(H)}{8 \Delta_{H}^{3}} \\
& +\frac{\left(6 \delta_{H}+3\right) R_{2}(H)+\left(12 \delta_{H}+3\right) R_{1}(H)+m_{2}}{8 \Delta_{H}^{3}} \\
& +\frac{n_{1} n_{2}\left(\delta_{G}+n_{2}\right)^{3}\left(\delta_{H}+1\right)^{3}}{\left(\Delta_{G}+\Delta_{H}+n_{2}-1\right)^{3}}
\end{aligned}
$$


with equality if and only if $G$ and $H$ are regular graphs.

Proof. The proof of the result is similar to this given in Theorem 3.1.

Corollary 3.1. Let $G$ be a k-regular graph of order $n_{1}$ and let $H$ be a r-regular graph of order $n_{2}$. Then

$$
A Z I(G \circ H)=\frac{k\left(k+n_{2}\right)^{6}}{16\left(k+n_{2}-1\right)^{3}}+\frac{r(r+1)^{6}}{16 r^{3}}+\frac{n_{1} n_{2}\left(k+n_{2}\right)^{3}(r+1)^{3}}{\left(k+r+n_{2}-1\right)^{3}} .
$$

\section{The Cartesian Product of Graphs}

The Cartesian product $G \times H$ of graphs $G$ and $H$ has the vertex set $V(G \times H)=$ $V(G) \times V(H)$ and $(u, x)(v, y)$ is an edge of $G \times H$ if $u v \in E(G)$ and $x=y$, or $u=v$ and $x y \in E(H)$. Obviously, $|V(G \times H)|=|V(G)||V(H)|$ and $|E(G \times H)|=$ $|E(G)||V(H)|+|V(G)||E(H)|$.

Theorem 4.1. Let $G$ be a graph of order $n_{1}$ and of size $m_{1}$ and let $H$ be a graph of order $n_{2}$ and of size $m_{2}$. Then

$$
\begin{aligned}
A Z I(G \times H) \leq & \frac{n_{2}\left(\Delta_{G}+\Delta_{H}-\delta_{H}-1\right)^{3} A Z I(G)+n_{1}\left(\Delta_{G}+\Delta_{H}-\delta_{G}-1\right)^{3} A Z I(H)}{\left(\Delta_{G}+\Delta_{H}-1\right)^{3}} \\
& +\frac{n_{2} \Delta_{H}^{3} \chi_{3}(G)+n_{2}\left(3 \Delta_{H}^{2} \Delta_{G}^{2}+3 \Delta_{H}^{4}\right) \chi_{2}(G)+3 n_{2} \Delta_{H}^{5} \chi_{1}(G)+\Delta_{G}^{6} m_{2}}{8\left(\delta_{G}+\delta_{H}-1\right)^{3}} \\
& +\frac{n_{1} \Delta_{G}^{3} \chi_{3}(H)+n_{1}\left(3 \Delta_{H}^{2} \Delta_{G}^{2}+3 \Delta_{G}^{4}\right) \chi_{2}(H)+3 n_{1} \Delta_{G}^{5} \chi_{1}(H)+\Delta_{H}^{6} m_{1}}{8\left(\delta_{G}+\delta_{H}-1\right)^{3}} \\
& +\frac{n_{2}\left(6 \Delta_{H} \Delta_{G}+3 \Delta_{H}^{2}\right) R_{2}(G)+n_{2}\left(12 \Delta_{H}^{3} \Delta_{G}+3 \Delta_{H}^{4}\right) R_{1}(G)}{8\left(\delta_{G}+\delta_{H}-1\right)^{3}} \\
& +\frac{n_{1}\left(6 \Delta_{H} \Delta_{G}+3 \Delta_{G}^{2}\right) R_{2}(H)+n_{1}\left(12 \Delta_{G}^{3} \Delta_{H}+3 \Delta_{G}^{4}\right) R_{1}(H)}{8\left(\delta_{G}+\delta_{H}-1\right)^{3}}
\end{aligned}
$$

with equality if and only if $G$ and $H$ are regular graphs.

Proof. By definition,

$$
A Z I(G \times H)=\sum_{(u, x)(v, y) \in E(G \times H)}\left(\frac{d_{G \times H}(u, x) d_{G \times H}(v, y)}{d_{G \times H}(u, x)+d_{G \times H}(v, y)-2}\right)^{3} .
$$

We partition the edges of $G \times H$ in to two subset $E_{1}$ and $E_{2}$, as follows:

$$
\begin{aligned}
& E_{1}=\{e=(u, x)(v, y) \mid u v \in E(G), x=y\}, \\
& E_{2}=\{e=(u, x)(v, y) \mid x y \in E(H), u=v\} .
\end{aligned}
$$

Let $e=(u, x)(v, x) \in E_{1}$. Then $d_{G \times H}(u, x)=d_{G}(u)+d_{H}(x)$ and $d_{G \times H}(v, x)=$ $d_{G}(v)+d_{H}(x)$. By used of proof of Theorem 2.1, we have

$$
\left(\frac{d_{G \times H}(u, x) d_{G \times H}(v, x)}{d_{G \times H}(u, x)+d_{G \times H}(v, x)-2}\right)^{3} \leq \frac{\left(\Delta_{G}+\Delta_{H}-\delta_{H}-1\right)^{3}}{\left(\Delta_{G}+\Delta_{H}-1\right)^{3}}\left(\frac{d_{G}(u) d_{G}(v)}{d_{G}(u)+d_{G}(v)-2}\right)^{3}
$$


Therefore,

$$
\begin{aligned}
& +\frac{\left.\Delta_{H}^{3}\left(d_{G}(u)+d_{G}(v)\right)^{3}\right)\left(d_{G}(u)+d_{G}(v)\right)^{2}}{8\left(\delta_{G}+\delta_{H}-1\right)^{3}} \\
& +\frac{\left(3 \Delta_{H}^{2} \Delta_{G}^{2}+3 \Delta_{H}^{4}\right)\left(d_{G}(u)+d_{G}(v)\right)^{2}}{8\left(\delta_{G}+\delta_{H}-1\right)^{3}} \\
& +\frac{3 \Delta_{H}^{5}\left(d_{G}(u)+d_{G}(v)\right)}{8\left(\delta_{G}+\delta_{H}-1\right)^{3}} \\
& +\frac{\left(6 \Delta_{H} \Delta_{G}+3 \Delta_{H}^{2}\right)\left(d_{G}(u) d_{G}(v)\right)^{2}}{8\left(\delta_{G}+\delta_{H}-1\right)^{3}} \\
& +\frac{\left(12 \Delta_{H}^{3} \Delta_{G}+3 \Delta_{H}^{4}\right) d_{G}(u) d_{G}(v)+\Delta_{H}^{6}}{8\left(\delta_{G}+\delta_{H}-1\right)^{3}} .
\end{aligned}
$$

$$
\begin{aligned}
& \sum_{(u, x)(v, x) \in E_{1}}\left(\frac{d_{G \times H}(u, x) d_{G \times H}(v, x)}{d_{G \times H}(u, x)+d_{G \times H}(v, x)-2}\right)^{3} \\
\leq & \frac{n_{2}\left(\Delta_{G}+\Delta_{H}-\delta_{H}-1\right)^{3} A Z I(G)}{\left(\Delta_{G}+\Delta_{H}-1\right)^{3}}+\frac{n_{2} \Delta_{H}^{3} \chi_{3}(G)+3 n_{2} \Delta_{H}^{5} \chi_{1}(G)}{8\left(\delta_{G}+\delta_{H}-1\right)^{3}} \\
+ & \frac{n_{2}\left(3 \Delta_{H}^{2} \Delta_{G}^{2}+3 \Delta_{H}^{4}\right) \chi_{2}(G)}{8\left(\delta_{G}+\delta_{H}-1\right)^{3}}+\frac{n_{2}\left(6 \Delta_{H} \Delta_{G}+3 \Delta_{H}^{2}\right) R_{2}(G)}{8\left(\delta_{G}+\delta_{H}-1\right)^{3}} \\
+ & \frac{n_{2}\left(12 \Delta_{H}^{3} \Delta_{G}+3 \Delta_{H}^{4}\right) R_{1}(G)+\Delta_{H}^{6} n_{2} m_{1}}{8\left(\delta_{G}+\delta_{H}-1\right)^{3}}
\end{aligned}
$$

Obviously, equality holds if and only if $\Delta_{G}=\delta_{G}$ and $\Delta_{H}=\delta_{H}$. Similarly,

$$
\begin{aligned}
\sum_{(u, x)(u, y) \in E_{2}}\left(\frac{d_{G \times H}(u, x) d_{G \times H}(u, y)}{d_{G \times H}(u, x)+d_{G \times H}(u, y)-2}\right)^{3} \leq & \frac{n_{1}\left(\Delta_{G}+\Delta_{H}-\delta_{G}-1\right)^{3} A Z I(H)}{\left(\Delta_{G}+\Delta_{H}-1\right)^{3}} \\
& +\frac{n_{1} \Delta_{G}^{3} \chi_{3}(H)+3 n_{1} \Delta_{G}^{5} \chi_{1}(H)}{8\left(\delta_{G}+\delta_{H}-1\right)^{3}} \\
& +\frac{n_{1}\left(3 \Delta_{H}^{2} \Delta_{G}^{2}+3 \Delta_{G}^{4}\right) \chi_{2}(H)}{8\left(\delta_{G}+\delta_{H}-1\right)^{3}} \\
& +\frac{n_{1}\left(6 \Delta_{H} \Delta_{G}+3 \Delta_{G}^{2}\right) R_{2}(H)}{8\left(\delta_{G}+\delta_{H}-1\right)^{3}} \\
& +\frac{n_{1}\left(12 \Delta_{G}^{3} \Delta_{H}+3 \Delta_{G}^{4}\right) R_{1}(H)+\Delta_{G}^{6} n_{1} m_{2}}{8\left(\delta_{G}+\delta_{H}-1\right)^{3}} .
\end{aligned}
$$

Equality holds if and only if $\Delta_{G}=\delta_{G}$ and $\Delta_{H}=\delta_{H}$. By Equations (4.1) and (4.2), we have:

$$
\begin{aligned}
A Z I(G \times H) \leq & \frac{n_{2}\left(\Delta_{G}+\Delta_{H}-\delta_{H}-1\right)^{3} A Z I(G)+n_{1}\left(\Delta_{G}+\Delta_{H}-\delta_{G}-1\right)^{3} A Z I(H)}{\left(\Delta_{G}+\Delta_{H}-1\right)^{3}} \\
& +\frac{n_{2} \Delta_{H}^{3} \chi_{3}(G)+n_{2}\left(3 \Delta_{H}^{2} \Delta_{G}^{2}+3 \Delta_{H}^{4}\right) \chi_{2}(G)+3 n_{2} \Delta_{H}^{5} \chi_{1}(G)+\Delta_{G}^{6} m_{2}}{8\left(\delta_{G}+\delta_{H}-1\right)^{3}}
\end{aligned}
$$




$$
\begin{aligned}
& +\frac{n_{1} \Delta_{G}^{3} \chi_{3}(H)+n_{1}\left(3 \Delta_{H}^{2} \Delta_{G}^{2}+3 \Delta_{G}^{4}\right) \chi_{2}(H)+3 n_{1} \Delta_{G}^{5} \chi_{1}(H)+\Delta_{H}^{6} m_{1}}{8\left(\delta_{G}+\delta_{H}-1\right)^{3}} \\
& +\frac{n_{2}\left(6 \Delta_{H} \Delta_{G}+3 \Delta_{H}^{2}\right) R_{2}(G)+n_{2}\left(12 \Delta_{H}^{3} \Delta_{G}+3 \Delta_{H}^{4}\right) R_{1}(G)}{8\left(\delta_{G}+\delta_{H}-1\right)^{3}} \\
& +\frac{n_{1}\left(6 \Delta_{H} \Delta_{G}+3 \Delta_{G}^{2}\right) R_{2}(H)+n_{1}\left(12 \Delta_{G}^{3} \Delta_{H}+3 \Delta_{G}^{4}\right) R_{1}(H)}{8\left(\delta_{G}+\delta_{H}-1\right)^{3}}
\end{aligned}
$$

with equality if and only if $G$ and $H$ are regular graphs.

Theorem 4.2. Let $G$ be a graph of order $n_{1}$ and of size $m_{1}$ and let $H$ be a graph of order $n_{2}$ and of size $m_{2}$. Then

$$
\begin{aligned}
A Z I(G \times H) \geq & \frac{n_{2}\left(\delta_{G}+\delta_{H}-\Delta_{H}-1\right)^{3} A Z I(G)+n_{1}\left(\delta_{G}+\delta_{H}-\Delta_{G}-1\right)^{3} A Z I(H)}{\left(\delta_{G}+\delta_{H}-1\right)^{3}} \\
& +\frac{n_{2} \delta_{H}^{3} \chi_{3}(G)+n_{2}\left(3 \delta_{H}^{2} \delta_{G}^{2}+3 \delta_{H}^{4}\right) \chi_{2}(G)+3 n_{2} \delta_{H}^{5} \chi_{1}(G)}{8\left(\Delta_{G}+\Delta_{H}-1\right)^{3}} \\
& +\frac{n_{2}\left(6 \delta_{H} \delta_{G}+3 \delta_{H}^{2}\right) R_{2}(G)+n_{2}\left(12 \delta_{H}^{3} \delta_{G}+3 \delta_{H}^{4}\right) R_{1}(G)+\delta_{H}^{6} m_{1}}{8\left(\Delta_{G}+\Delta_{H}-1\right)^{3}} \\
& +\frac{n_{1} \delta_{G}^{3} \chi_{3}(H)+n_{1}\left(3 \delta_{H}^{2} \delta_{G}^{2}+3 \delta_{G}^{4}\right) \chi_{2}(H)+3 n_{1} \delta_{G}^{5} \chi_{1}(H)}{8\left(\Delta_{G}+\Delta_{H}-1\right)^{3}} \\
& +\frac{n_{1}\left(6 \delta_{H} \delta_{G}+3 \delta_{G}^{2}\right) R_{2}(H)+n_{1}\left(12 \delta_{G}^{3} \delta_{H}+3 \delta_{G}^{4}\right) R_{1}(H)+\delta_{G}^{6} m_{2}}{8\left(\Delta_{G}+\Delta_{H}-1\right)^{3}},
\end{aligned}
$$

with equality if and only if $G$ and $H$ are regular graphs.

Proof. Using an argument similar to that described in proof of Theorem 4.1, we obtained the result.

Corollary 4.1. Let $G$ be a $k$-regular graph of order $n_{1}$ and let $H$ be a $r$-regular graph of order $n_{2}$. Then $A Z I(G \times H)=\frac{n_{1} n_{2}(k+r)^{7}}{16(k+r-1)^{3}}$.

\section{The Composition Product of Graphs}

The composition $G[H]$ of graphs $G$ and $H$ has the vertex set $V(G[H])=V(G) \times$ $V(H)$ and $(u, x)(v, y)$ is an edge of $G[H]$ if $(u v \in E(G))$ or $(x y \in E(H)$ and $u=v)$. Obviously, $|V(G[H])|=|V(G)||V(H)|$ and $|E(G[H])|=|E(G)||V(H)|^{2}+$ $|E(H)||V(G)|$.

Theorem 5.1. Let $G$ be a graph of order $n_{1}$ and of size $m_{1}$ and let $H$ be a graph of order $n_{2}$ and of size $m_{2}$. Then

$$
\begin{aligned}
& A Z I(G[H]) \\
\leq & \frac{n_{2}^{5}\left(n_{2} \Delta_{G}+\Delta_{H}-\delta_{H}-n_{2}\right)^{3} A Z I(G)+n_{1}\left(\Delta_{H}+n_{2} \Delta_{G}-n_{2} \delta_{G}-1\right)^{3} A Z I(H)}{\left(n_{2} \Delta_{G}+\Delta_{H}-1\right)^{3}}
\end{aligned}
$$




$$
\begin{aligned}
& +\frac{n_{2}^{5} \Delta_{H}^{3} \chi_{3}(G)+n_{2}^{2}\left(3 n_{2}^{4} \Delta_{H}^{2} \Delta_{G}^{2}+3 n_{2}^{2} \Delta_{H}^{4}\right) \chi_{2}(G)+3 n_{2}^{3} \Delta_{H}^{5} \chi_{1}(G)}{8\left(n_{2} \delta_{G}+\delta_{H}-1\right)^{3}} \\
& +\frac{n_{1} n_{2}^{3} \Delta_{G}^{3} \chi_{3}(H)+n_{1}\left(3 n_{2}^{2} \Delta_{H}^{2} \Delta_{G}^{2}+3 n_{2}^{4} \Delta_{G}^{4}\right) \chi_{2}(H)+3 n_{1} n_{2}^{5} \Delta_{G}^{5} \chi_{1}(H)}{8\left(n_{2} \delta_{G}+\delta_{H}-1\right)^{3}} \\
& +\frac{n_{2}^{2}\left(6 n_{2}^{5} \Delta_{H} \Delta_{G}+3 n_{2}^{4} \Delta_{H}^{2}\right) R_{2}(G)+n_{2}^{2}\left(12 n_{2}^{3} \Delta_{H}^{3} \Delta_{G}+3 n_{2}^{2} \Delta_{H}^{4}\right) R_{1}(G)+n_{2}^{2} m_{1} \Delta_{H}^{6}}{8\left(n_{2} \delta_{G}+\delta_{H}-1\right)^{3}} \\
& +\frac{n_{1}\left(6 n_{2} \Delta_{H} \Delta_{G}+3 n_{2}^{2} \Delta_{G}^{2}\right) R_{2}(H)+n_{1}\left(12 n_{2}^{3} \Delta_{G}^{3} \Delta_{H}+3 n_{2}^{4} \Delta_{G}^{4}\right) R_{1}(H)+n_{1} m_{2} n_{2}^{6} \Delta_{G}^{6}}{8\left(n_{2} \delta_{G}+\delta_{H}-1\right)^{3}},
\end{aligned}
$$

with equality if and only if $G$ and $H$ are regular graphs.

Proof. We partition the edges of $G[H]$ in to two subset $E_{1}$ and $E_{2}$, as follows:

$$
\begin{aligned}
& E_{1}=\{e=(u, x)(v, y) \mid u v \in E(G)\}, \\
& E_{2}=\{e=(u, x)(v, y) \mid x y \in E(H), u=v\} .
\end{aligned}
$$

Let $e=(u, x)(v, y) \in E_{1}$. Then $d_{G[H]}(u, x)=n_{2} d_{G}(u)+d_{H}(x)$ and $d_{G[H]}(v, y)=$ $n_{2} d_{G}(v)+d_{H}(y)$. By used of proof of Theorem 2.1, we have,

$$
\begin{aligned}
\left(\frac{d_{G[H]}(u, x) d_{G[H]}(v, y)}{d_{G[H]}(u, x)+d_{G[H]}(v, y)-2}\right)^{3} \leq & \frac{n_{2}^{5}\left(n_{2} \Delta_{G}+\Delta_{H}-\delta_{H}-n_{2}\right)^{3}}{\left(n_{2} \Delta_{G}+\Delta_{H}-1\right)^{3}}\left(\frac{d_{G}(u) d_{G}(v)}{d_{G}(u)+d_{G}(v)-2}\right)^{3} \\
& +\frac{n_{2}^{3} \Delta_{H}^{3}\left(d_{G}(u)+d_{G}(v)\right)^{3}+3 n_{2} \Delta_{H}^{5}\left(d_{G}(u)+d_{G}(v)\right)}{8\left(n_{2} \delta_{G}+\delta_{H}-1\right)^{3}} \\
& +\frac{\left(3 n_{2}^{4} \Delta_{H}^{2} \Delta_{G}^{2}+3 n_{2}^{2} \Delta_{H}^{4}\right)\left(d_{G}(u)+d_{G}(v)\right)^{2}}{8\left(n_{2} \delta_{G}+\delta_{H}-1\right)^{3}} \\
& +\frac{\left(6 n_{2}^{5} \Delta_{H} \Delta_{G}+3 n_{2}^{4} \Delta_{H}^{2}\right)\left(d_{G}(u) d_{G}(v)\right)^{2}}{8\left(n_{2} \delta_{G}+\delta_{H}-1\right)^{3}} \\
& +\frac{\left(12 n_{2}^{3} \Delta_{H}^{3} \Delta_{G}+3 n_{2}^{2} \Delta_{H}^{4}\right) d_{G}(u) d_{G}(v)+\Delta_{H}^{6}}{8\left(n_{2} \delta_{G}+\delta_{H}-1\right)^{3}} .
\end{aligned}
$$

Therefore,

$$
\begin{aligned}
\sum_{(u, x)(v, y) \in E_{1}}\left(\frac{d_{G[H]}(u, x) d_{G[H]}(v, x)}{d_{G[H]}(u, x)+d_{G[H]}(v, x)-2}\right)^{3} \leq & \frac{n_{2}^{5}\left(n_{2} \Delta_{G}+\Delta_{H}-\delta_{H}-n_{2}\right)^{3} A Z I(G)}{\left(n_{2} \Delta_{G}+\Delta_{H}-1\right)^{3}} \\
& +\frac{n_{2}^{2}\left(6 n_{2}^{5} \Delta_{H} \Delta_{G}+3 n_{2}^{4} \Delta_{H}^{2}\right) R_{2}(G)}{8\left(n_{2} \delta_{G}+\delta_{H}-1\right)^{3}} \\
& +\frac{n_{2}^{2}\left(3 n_{2}^{4} \Delta_{H}^{2} \Delta_{G}^{2}+3 n_{2}^{2} \Delta_{H}^{4}\right) \chi_{2}(G)}{8\left(n_{2} \delta_{G}+\delta_{H}-1\right)^{3}} \\
& +\frac{n_{2}^{5} \Delta_{H}^{3} \chi_{3}(G)}{8\left(n_{2} \delta_{G}+\delta_{H}-1\right)^{3}}+\frac{3 n_{2}^{3} \Delta_{H}^{5} \chi_{1}(G)}{8\left(n_{2} \delta_{G}+\delta_{H}-1\right)^{3}} \\
& +\frac{n_{2}^{2}\left(12 n_{2}^{3} \Delta_{H}^{3} \Delta_{G}+3 n_{2}^{2} \Delta_{H}^{4}\right) R_{1}(G)}{8\left(n_{2} \delta_{G}+\delta_{H}-1\right)^{3}}
\end{aligned}
$$




$$
+\frac{n_{2}^{2} m_{1} \Delta_{H}^{6}}{8\left(n_{2} \delta_{G}+\delta_{H}-1\right)^{3}} .
$$

Obviously, equality holds if and only if $\Delta_{G}=\delta_{G}$ and $\Delta_{H}=\delta_{H}$. Similarly,

$$
\begin{aligned}
\sum_{(u, x)(u, y) \in E_{2}}\left(\frac{d_{G[H]}(u, x) d_{G[H]}(u, y)}{d_{G[H]}(u, x)+d_{G[H]}(u, y)-2}\right)^{3} \leq & \frac{n_{1}\left(\Delta_{H}+n_{2} \Delta_{G}-n_{2} \delta_{G}-1\right)^{3} A Z I(H)}{\left(n_{2} \Delta_{G}+\Delta_{H}-1\right)^{3}} \\
& +\frac{n_{1}\left(6 n_{2} \Delta_{H} \Delta_{G}+3 n_{2}^{2} \Delta_{G}^{2}\right) R_{2}(H)}{8\left(n_{2} \delta_{G}+\delta_{H}-1\right)^{3}} \\
& +\frac{n_{1}\left(3 n_{2}^{2} \Delta_{H}^{2} \Delta_{G}^{2}+3 n_{2}^{4} \Delta_{G}^{4}\right) \chi_{2}(H)}{8\left(n_{2} \delta_{G}+\delta_{H}-1\right)^{3}} \\
& +\frac{n_{1} n_{2}^{3} \Delta_{G}^{3} \chi_{3}(H)}{8\left(\delta_{G}+\delta_{H}-1\right)^{3}+\frac{3 n_{1} n_{2}^{5} \Delta_{G}^{5} \chi_{1}(H)}{8\left(n_{2} \delta_{G}+\delta_{H}-1\right)^{3}}} \\
& +\frac{n_{1}\left(12 n_{2}^{3} \Delta_{G}^{3} \Delta_{H}+3 n_{2}^{4} \Delta_{G}^{4}\right) R_{1}(H)}{8\left(n_{2} \delta_{G}+\delta_{H}-1\right)^{3}} \\
& +\frac{n_{1} m_{2} n_{2}^{6} \Delta_{G}^{6}}{8\left(n_{2} \delta_{G}+\delta_{H}-1\right)^{3}} .
\end{aligned}
$$

Equality holds if and only if $\Delta_{G}=\delta_{G}$ and $\Delta_{H}=\delta_{H}$. By Equations (5.1) and (5.2), we have:

$$
\begin{aligned}
& A Z I(G[H]) \\
\leq & \frac{n_{2}^{5}\left(n_{2} \Delta_{G}+\Delta_{H}-\delta_{H}-n_{2}\right)^{3} A Z I(G)+n_{1}\left(\Delta_{H}+n_{2} \Delta_{G}-n_{2} \delta_{G}-1\right)^{3} A Z I(H)}{\left(n_{2} \Delta_{G}+\Delta_{H}-1\right)^{3}} \\
& +\frac{n_{2}^{5} \Delta_{H}^{3} \chi_{3}(G)+n_{2}^{2}\left(3 n_{2}^{4} \Delta_{H}^{2} \Delta_{G}^{2}+3 n_{2}^{2} \Delta_{H}^{4}\right) \chi_{2}(G)+3 n_{2}^{3} \Delta_{H}^{5} \chi_{1}(G)}{8\left(n_{2} \delta_{G}+\delta_{H}-1\right)^{3}} \\
& +\frac{n_{1} n_{2}^{3} \Delta_{G}^{3} \chi_{3}(H)+n_{1}\left(3 n_{2}^{2} \Delta_{H}^{2} \Delta_{G}^{2}+3 n_{2}^{4} \Delta_{G}^{4}\right) \chi_{2}(H)+3 n_{1} n_{2}^{5} \Delta_{G}^{5} \chi_{1}(H)}{8\left(n_{2} \delta_{G}+\delta_{H}-1\right)^{3}} \\
& +\frac{n_{2}^{2}\left(6 n_{2}^{5} \Delta_{H} \Delta_{G}+3 n_{2}^{4} \Delta_{H}^{2}\right) R_{2}(G)+n_{2}^{2}\left(12 n_{2}^{3} \Delta_{H}^{3} \Delta_{G}+3 n_{2}^{2} \Delta_{H}^{4}\right) R_{1}(G)+n_{2}^{2} m_{1} \Delta_{H}^{6}}{8\left(n_{2} \delta_{G}+\delta_{H}-1\right)^{3}} \\
& +\frac{n_{1}\left(6 n_{2} \Delta_{H} \Delta_{G}+3 n_{2}^{2} \Delta_{G}^{2}\right) R_{2}(H)+n_{1}\left(12 n_{2}^{3} \Delta_{G}^{3} \Delta_{H}+3 n_{2}^{4} \Delta_{G}^{4}\right) R_{1}(H)+n_{1} m_{2} n_{2}^{6} \Delta_{G}^{6}}{8\left(n_{2} \delta_{G}+\delta_{H}-1\right)^{3}},
\end{aligned}
$$

with equality if and only if $G$ and $H$ are regular graphs.

Theorem 5.2. Let $G$ be a graph of order $n_{1}$ and of size $m_{1}$ and let $H$ be a graph of order $n_{2}$ and of size $m_{2}$. Then

$$
\begin{aligned}
& A Z I(G[H]) \\
\geq & \frac{n_{2}^{5}\left(n_{2} \delta_{G}+\delta_{H}-\Delta_{H}-n_{2}\right)^{3} A Z I(G)+n_{1}\left(\delta_{H}+n_{2} \delta_{G}-n_{2} \Delta_{G}-1\right)^{3} A Z I(H)}{\left(n_{2} \delta_{G}+\delta_{H}-1\right)^{3}}
\end{aligned}
$$




$$
\begin{aligned}
& +\frac{n_{2}^{5} \delta_{H}^{3} \chi_{3}(G)+n_{2}^{2}\left(3 n_{2}^{4} \delta_{H}^{2} \delta_{G}^{2}+3 n_{2}^{2} \delta_{H}^{4}\right) \chi_{2}(G)+3 n_{2}^{3} \delta_{H}^{5} \chi_{1}(G)}{8\left(n_{2} \Delta_{G}+\Delta_{H}-1\right)^{3}} \\
& +\frac{n_{1} n_{2}^{3} \delta_{G}^{3} \chi_{3}(H)+n_{1}\left(3 n_{2}^{2} \delta_{H}^{2} \delta_{G}^{2}+3 n_{2}^{4} \delta_{G}^{4}\right) \chi_{2}(H)+3 n_{1} n_{2}^{5} \delta_{G}^{5} \chi_{1}(H)}{8\left(n_{2} \Delta_{G}+\Delta_{H}-1\right)^{3}} \\
& +\frac{n_{2}^{2}\left(6 n_{2}^{5} \delta_{H} \delta_{G}+3 n_{2}^{4} \delta_{H}^{2}\right) R_{2}(G)+n_{2}^{2}\left(12 n_{2}^{3} \delta_{H}^{3} \delta_{G}+3 n_{2}^{2} \delta_{H}^{4}\right) R_{1}(G)+n_{2}^{2} m_{1} \delta_{H}^{6}}{8\left(n_{2} \Delta_{G}+\Delta_{H}-1\right)^{3}} \\
& +\frac{n_{1}\left(6 n_{2} \delta_{H} \delta_{G}+3 n_{2}^{2} \delta_{G}^{2}\right) R_{2}(H)+n_{1}\left(12 n_{2}^{3} \delta_{G}^{3} \delta_{H}+3 n_{2}^{4} \delta_{G}^{4}\right) R_{1}(H)+n_{1} m_{2} n_{2}^{6} \delta_{G}^{6}}{8\left(n_{2} \Delta_{G}+\Delta_{H}-1\right)^{3}},
\end{aligned}
$$

with equality if and only if $G$ and $H$ are regular graphs.

Proof. The proof of the result is similar to this given in Theorem 5.1.

Corollary 5.1. Let $G$ be a k-regular graph of order $n_{1}$ and let $H$ be a r-regular graph of order $n_{2}$. Then $A Z I(G[H])=\frac{n_{1} n_{2}\left(n_{2} k+r\right)^{7}}{16\left(n_{2} k+r-1\right)^{3}}$.

\section{REFERENCES}

[1] A. Ali, Z. Raza and A. A. Bhatti, On the augmented Zagreb index, Kuwait J. Sci. 43 (2016), 48-63.

[2] H. Aram and N. Dehgardi, Reformulated F-index of graph operations, Commun. Comb. Optim. 2 (2017), 1-12.

[3] H. Aram, N. Dehgardi and A. Khodkar, The third ABC index of graph products, Bull. Int. Combin. Math. Appl. 78 (2016), 69-82.

[4] M. Arezoomand and B. Taeri, Zagreb indices of the generalized hierarchical product of graphs, MATCH Commun. Math. Comput. Chem. 69 (2013), 131-140.

[5] A. R. Ashrafi, T. Došlić and A. Hamzeh, The Zagreb coindices of graph operations, Discrete Appl. Math. 158 (2010), 1571-1578.

[6] M. Azari, Sharp lower bounds on the Narumi-Katayama index of graph operations, Appl. Math. Comput. 239 (2014), 409-421.

[7] M. Azari and A. Iranmanesh, Chemical graphs constructed from rooted product and their Zagreb indices, MATCH Commun. Math. Comput. Chem. 70 (2013), 901-919.

[8] M. Azari and A. Iranmanesh, Some inequalities for the multiplicative sum Zagreb index of graph operations, J. Math. Inequal. 9 (2015), 727-738.

[9] B. Bollobás and P. Erdós, Graphs of extremal weights, Ars Combin. 50 (1998), 225-233.

[10] J. A. Bondy and U. S. R. Murty, Graph Theory, Graduate Texts in Mathematics 244, SpringerVerlag, London, 2008.

[11] K. C. Das, A. Yurttas, M. Togan, A. S. Cevik and I. N. Cangül, The multiplicative Zagreb indices of graph operations, J. Inequal. Appl. 90, (2013), 1-14.

[12] N. Dehgardi, A note on revised Szeged index of graph operations, Iranian J. Math. Chem. 9(1) (2018), 57-63.

[13] K. Fathalikhani, H. Faramarzi and H. Yousefi-Azari, Total eccentricity of some graph operations, Electron. Notes in Discrete Math. 45 (2014), 125-131.

[14] G. A. Fath-Tabar, B. Vaez-Zadah, A. R. Ashrafi and A. Graovac, Some inequalities for the atom-bond connectivity index of graph operations, Discrete Appl. Math. 159 (2011), 1323-1330.

[15] B. Furtula, A. Graovac and D. Vukičević, Augmented Zagreb index, J. Math. Chem. 48 (2010), $370-380$

[16] B. Furtula, I. Gutman and M. Dehmer, On structure-sensitivity of degree-based topological indices, Appl. Math. Comput. 219(1) (2013), 8973-8978. 
[17] S. Hossein-Zadeh, A. Hamzeh and A. R. Ashrafi, Wiener-type invariants of some graph operations, Filomat 23 (2009), 103-113.

[18] Y. Huang, B. Liu and L. Gan, Augmented Zagreb index of connected graphs, MATCH Commun. Math. Comput. Chem. 67 (2012), 483-494.

[19] M. H. Khalifeh, H. Yusefi Azari and A. R. Ashrafi, The first and second Zagreb indices of some graph operations, Discrete Appl. Math. 157 (2009), 804-811.

[20] K. Pattabiraman and P. Paulraja, Harary index of product graph, Discuss. Math. Graph Theory 35 (2015) 17-33.

[21] M. Randić, On characterization of molecular branching, J. Amer. Chem. Soc. 97 (1975), 66096615 .

[22] G. Su and L. Xu, Topological indices of the line graph of subdivision graphs and their Schurbounds, Appl. Math. Comput. 253 (2015), 395-401.

[23] I. Tomescu, 2-Connected graphs with minimum general sum-connectivity index, Discrete Appl. Math. 178 (2014), 135-141.

[24] D. Wang, Y. Huang and B. Liu, Bounds on augmented Zagreb index, MATCH Commun. Math. Comput. Chem. 68 (2012), 209-216.

[25] Z. Yarahmadi and A. R. Ashrafi, The Szeged, vertex PI, first and second Zagreb indices of corona product of graphs, Filomat 26 (2012), 467-472.

[26] Y-N. Yeh and I. Gutman, On the sum of all distances in composite graphs, Discrete Math. 135 (1994), 17-20.

[27] F. Zhan, Y. Qiao and J. Cai, Unicyclic and bicyclic graphs with minimal augmented Zagreb index, J. Inequal. Appl. 126, (2015), 1-12.

[28] B. Zhou and N. Trinajstić, On a novel connectivity index, J. Math. Chem. 46 (2009), 1252-1270.

[29] B. Zhou and N. Trinajstić, On general sum-connectivity index, J. Math. Chem. 47 (2010), $210-218$.

${ }^{1}$ Department of Mathematics and Computer Science,

SiRJAN UNIVERSITY OF TECHNOLOGY,

SIRJAN, I.R. IRAN

Email address: n.dehgardi@sirjantech.ac.ir

${ }^{2}$ Department of Mathematics,

Gareziaeddin Center,Khoy Branch,Islamic Azad University,

KHOY, IRAN

Email address: hamideh.aram@gmail.com

*Corresponding AUthor 